Review Articles

\title{
Scope and Perspectives of New TB Drugs and Vaccines
}

\author{
Jaishree Meena and Laxman S. Meena \\ CSIR-Institute of Genomics and Integrative Biology, Council of Scientific and Industrial Research, \\ Mall Road, Delhi-110007 India
}

\author{
Article history \\ Received: 09-04-2015 \\ Revised: $15-05-2015$ \\ Accepted: 29-08-2015 \\ Corresponding Author: \\ Laxman S. Meena \\ CSIR-Institute of Genomics \\ and Integrative Biology, \\ Council of Scientific and \\ Industrial Research, Mall Road, \\ Delhi-110007 India \\ E-mail: meena@igib.res.in
}

\begin{abstract}
Tuberculosis remains a major threatening infectious disease claiming millions of lives every year; hence increasing the global burden of TB disease. Co-infection with human immuno-deficiency virus and emergence of multi-drug resistant tuberculosis, extremely drug resistant tuberculosis and totally drug resistant tuberculosis enhances deadly effect of tuberculosis. To eliminate TB disease from human population several partnerships are working together like Stop TB Partnership, TB Alliance, Tuberculosis vaccine initiative, Global Alliance for TB Drug Development and many other partners. Till now almost 10 drugs and 11 vaccines are under various clinical trials. Anti-Tuberculosis drugs like bedaquiline and clofazimine are being developed for treatment of tuberculosis as well as for treating MDR-TB, XDR-TB and TDR-TB but till now, no drugs are available for treatment of TDR-TB. PA-824 and SQ-109 showed their potent activity against tuberculosis and thought to reduce the treatment duration in infected patients. Several vaccines are also being developed for enhancing the effect of Bacillus-CalmetteGuerin as well for replacing it. New anti-tuberculosis drugs offer hopes of shortened treatment regimens for drug susceptible, drug resistant and latent infection. The quest for better vaccines for controlling tuberculosis among human population in future continues.
\end{abstract}

Keywords: Mycobacterium Tuberculosis, MDR-TB, XDR-TB, TDRTB, Drug and Vaccines

\section{Introduction}

Tuberculosis is a common, contagious infectious disease and is a major cause of deaths worldwide. In 2011, 8.7 million new cases of Tuberculosis (TB) were reported including $13 \%$ co-infected with Human Immuno-Deficiency Virus (HIV). HIV co-infection weakens the immune system of patient and hence there can be chances of opportunistic infections caused by mycobacterial species leading to active TB disease (WHO, 2012; Meena et al., 2008; Meena and Rajni, 2011). Along with acquired immune deficiency syndrome (AIDS), Multi-Drug Resistant Tuberculosis (MDR-TB), extremely Drug Resistant Tuberculosis (XDR-TB) (Singh et al., 2011) and Totally Drug Resistant Tuberculosis (TDR-TB) became major threat for TB treatment. Till today, more than 20 anti-TB drugs are available for use and these were developed a few years ago. First line drugs are mainly bactericidal; these include isoniazid, rifampicin, streptomycin, ethambutol, pyrazinamide and fluoroquinolone. Second line drugs are bacteriostatic and these are more toxic and less efficient than first line drugs. Second line drugs are the vast category of drugs generally used according to effectiveness, experience of use, dose and drug class. Currently, at least 10 chemical compounds are in various stages of clinical trials for TB treatment. These include the drugs for treating MDR-TB, XDR-TB and TDR-TB patients as well as patients having HIV co-infection. Rifapentine, linezolid, moxafloxacin are existing drugs which are under re-evaluation or re-development. SQ109, PNU-100480, PA-824, AZD-5847, Sirturo and Delamanid are some of the drugs which are being developed specifically for TB. Sirturo is an anti-TB drug developed by Janssen pharmaceutical, has got approval in December, 2012 as MDR-TB drug. Another drug Delamanid is a potential drug for treating drug resistant as well as drug susceptible TB. For treating MDR-TB including XDR-TB strains. AZD-5847, rifapentine, PA824 are some anti-TB drugs which are being developed and proved to be effective in TB Treatment (TB Drugs; http://www.tbfacts.org/tb-drugs.html). But the emergence of TDR-TB has led to the difficulties of researchers. Totally Drug Resistant Tuberculosis was described for the TB strains that are resistant to all first line and second line drugs. Cases of TDR-TB were 
found in Iran, Italy and India. More recently few cases of TDR-TB are also reported in South Africa as well by Centers of Diseases Control and Preventions. TDR-TB raised various questions to researchers like hostmicrobe's interaction, protection for laboratory personnels who treat TDR-TB patients and most important, creating new drugs which are effective against such TB-strains on the basis of cell wall, size and shape of bacilli (Velayati et al., 2013; Meena et al., 2013; Rajni and Meena, 2010; Meena and Rajni, 2010; Rajni et al., 2011). For the prevention of this highly infectious disease many vaccines are also being developed. BCG (Bacillus- Calmette-Guerin) vaccine is almost 100 years old. BCG protects against severe forms of TB in children but its efficiency is variable in preventing pulmonary $\mathrm{TB}$ in adults. BCG is not recommended for infants infected with HIV due to risk of disseminated BCG disease. Several studies are being carried out to develop TB vaccines as alternatives to BCG as well as for enhancing the effect of BCG. There are 12 new vaccines in pipeline in which 11 are for TB prevention and 1 is being developed to be used as immunotherapeutic agent (to improve responsiveness to chemotherapy). MVA85A is an attenuated vaccinia-vectored vaccine candidate designed for various human groups. It is in advanced clinical testing among all existing vaccine candidates and showed appreciable results in adults but had failed in case of infants. AERAS-402 / Crucell Ad35 are designed as a booster for infants, adolescents and adults. AERAS-402 is being tested in phase $2 b$ trials in Kenya, Mozambique and South Africa. M72 and Hybrid-1 are 2 different subunit vaccines in phase-2a trials in Europe and Africa. VPM-1002, RUTI, Hyvac-4, ID93 are some of the promising vaccines underdevelopment against TB (WHO, 2012).

\section{Drugs Under Development for Tuberculosis Treatment}

\section{Rifapentine}

Rifapentine is a semi-synthetic anti-mycobacterial drug which was approved by Food and Drug Administration in June, 1998. Rifapentine is 3-[(4cyclopentyl-1-piperazinyl) imino-methyl] rifamycin SV derivative and marketed under the trade name Priftin by Sanofi Aventis (Arioli et al., 1981) (Table 1). It acts against gram-positive and gram- negative bacteria as well as Mycobacterium Tuberculosis (MTB). It has inhibitory effect against deoxyribose nucleic acid dependent ribonucleic acid polymerase and also prevents ribonucleic acid transcription. Rifapentine is a drug under re-evaluation and has entered phase-2 clinical trials. Minimum Inhibitory Concentration (MIC) of Rifapentine is $0.06 \mu \mathrm{g} / \mathrm{ml}$ for mycobacteria. The 5 days $/$ week administration of $10 \quad \mathrm{mg} / \mathrm{kg}$ rifapentine+isoniazid+pyrazinamide for about 3 months in mouse was found to be satisfactory in curing the disease, but the results of clinical trials were not found to be favorable. This contradictions and experimental findings have led to further investigation (Grosset et al., 2012).

\section{Clofazimine}

Clofazimine is a fat-soluble riminophenazine dye which goes under the trade name Lamprene for treatment of leprosy. Barry et al. (1957) in 1954 developed clofazimine as an anti-TB drug and named it B663 (Barry et al., 1956; 1957). Clofazimine acts by binding to guanine bases of bacterial DNA and hence blocking the template function of DNA. It also inhibits bacterial proliferation by increasing activity of bacterial phospholipase resulting in release and accumulation of lysophospholipids which are toxic and anti-proliferative in nature and are possible mediator of clofazimine against bacteria. It is an existing drug under reevaluation. Its MIC is in the range of $0.6-1.2 \mu \mathrm{g} / \mathrm{ml}$ for MTB (Grumbach, 1960; Yawalkar and Vischer, 1979). One of the clinical reports with MDR-TB patients treated with combination regimens including clofazimine was done from January 2008 to March 2011, in Shanghai Pulmonary Hospital and has shown noticeable effect of clofazimine in TB treatment. No serious side-effects and toxicities were reported for clofazimine in cohort. Out of 39 MDR-TB patients, 15 patients successfully completed treatment and 22 patients achieved culture conversion (Xu et al., 2012). Inspite of its side-effects like skin discoloration and accumulation of crystals in tissue, clofazimine has been included in the drugs being considered for treatment of MDR-TB/XDR-TB (Table 1), due to its favorable results in a short, 9 month combined drug regimen for treatment of MDR-TB (Van et al., 2010). Encouraging results in murine models as per the details described by various groups (Barry et al., 1956; 1957; Grumbach, 1960) and MDRTB/XDR-TB patient outcomes treated with Clofazimine containing regimens are enough to support further laboratory and clinical studies on this drug.

\section{Moxifloxacin}

Moxifloxacin or BA $12-8039$ is a fourth generation synthetic drug developed by Bayer AG and marketed under the trade names Avelox, Avalox and Avalon for oral treatment. It is basically a fluoroquinolone antibacterial agent which interferes with the bacterial survival by binding to the DNA gyrase and topoisomerase IV, the necessary enzymes for replication, translation, repair and recombination of deoxyribonucleic acid. Moxifloxacin is a broad spectrum antibiotic used for a number of infections including respiratory tract infection, cellulitis, anthrax, meningitis, TB. It has a MIC of $0.5 \mu \mathrm{g} / \mathrm{ml}$ for MTB and has an equivalent anti-bacterial activity as that of isoniazid against MTB both in-vitro and in mice models of TB (Nuermberger et al., 2004; Ji et al., 1998; Yoshimatsu et al., 2002; Miyazaki et al., 1999; Lenaerts et al., 2003). In standard 4-drug regimen for drug susceptible cases of TB, the use of moxafloxacin 
instead of ethambutol was thought to shorten the treatment duration. To test this hypothesis, first phase II study was conducted in KwaZulu Natal, South Africa in June, 2004 among 217 patients (Rustomjee et al., 2008). A second phase II study was also conducted in Rio de Janeiro, Brazil (Conde et al., 2009) aiming at the same goal from October 2004 to March 2007 among 147 patients. The third phase II study was conducted by Tuberculosis Trials Consortium (TBTC). It was a multicenter study in 328 patients to evaluate the advantages of moxafloxacin $(400 \mathrm{mg})$ substitution for isoniazid $(300 \mathrm{mg}$ ) in standard drug regimen against pulmonary TB (Dorman et al., 2009). The results were not so encouraging when moxafloxacin was substituted for isoniazid while results were significantly beneficial when it was substituted for ethambutol (Rustomjee et al., 2008; Conde et al., 2009; Dorman et al., 2009). Currently this drug is in phase III clinical trials and is used in combined drug regimens for MDR-TB (TB Online, Moxifloxacin; www.tbonline.info/posts/2011/8/23/moxifloxacin).

\section{TMC-207}

TMC-207 or Sirturo (brand name) is a diarylquinoline R207910, anti-TB drug discovered by Andries et al. (2005) at Janssen Pharmaceutical. It is also called as Bedaquiline (Fig. 1). It is the first new molecule specifically approved by Food and Drug Administration (FDA) to treat MDR-TB and XDR-TB in 40 years as part of fast-track approval. This drug was introduced for the first time in 2004 at Interscience Conference on Antimicrobial Agents and Chemotherapy (ICAAC) meeting. Bedaquiline acts on proton pump for ATP synthase, inhibiting ATP synthesis (Cole and Alzari, 2005; Koul et al., 2007). Its MIC is $0.06 \mu \mathrm{g} / \mathrm{ml}$ for MTB (Andries et al., 2005) and it is in phase III trials. The results of phase I, II and IIb trials showed, it to be a safe and effective drug (Diacon et al., 2007). According to latest CDC Guidelines, bedaquiline should not be used alone and should be used only in combination with at least 3 other drugs (a 4-drug regimen) to which the patient's MDR-TB isolate has been shown to be susceptible in vitro. Administration of Bedaquiline in MDR-TB treatment regimen increased the sputum culture conversion from $9 \%$ to $48 \%$ and reduced the duration it takes for sputum negative conversion by $58 \%$ (Fact Sheet: Why Bedaquiline (TMC207) should be prioritized for drug-resistant TB patients in South Africa. www.msfaccess.org/.../fact-sheet-why-bedaquiline-

tmc207-should-be-prioritised-drug-resistant-tb-patientssouth). The drug Bedaquiline has side effects too; this drug carries black-box warming which means it can cause a potentially fatal abnormal heart rhythm (Voelker, 2013). The role of Bedaquiline will be decided on the basis of current and future studies, in shortening the treatment regimen for drug sensitive TB, latent $T B$ infection and effective regimen for MDR-TB.

\section{$P A-824$}

PA-824 (developed by TB Alliance) is a bicyclic nitroimidazole pro-drug; that is inactive chemical derivative which will get converted into active pharmacological agent inside bacterium. It is a potential, experimental drug, effective against protozoa and anaerobic bacteria (Grosset et al., 2012). PA-824 showed a mixed effect both on genes responsive to both cell wall inhibition (may inhibit ketomycolates) like INH and respiratory poisoning like cyanide. During enzymatic nitro-reduction, it donates nitric oxide which causes poisoning in respiratory apparatus. It acts as an antibacterial agent against both replicating \& hypoxic, nonreplicating bacilli. Its MIC is $0.125 \mu \mathrm{g} / \mathrm{ml}$ for drug susceptible and drug resistant strain of MTB (Stover et al., 2000; Tyagi et al., 2005) (Table 1). It gives promising results in murine models of TB by showing bactericidal activity during both initial and continuation phase of TB treatment (Tyagi et al., 2005; Lenaerts et al., 2005; Nuermberger et al., 2006; Tasneen et al., 2008). PA-824 contributes to an admirable sterilizing regimen (Nuermberger et al., 2008), when given daily with moxafloxacin and pyrazinamide as combination therapy. It is believed that PA-824 can shorten the treatment duration for drug susceptible as well as MDR-TB in humans. Currently, PA-824 is in phase-2 trials and further studies are needed to increase the efficacy and to eliminate its dependency on Pyrazinamide (because MDR-TB strains are likely to be resistant to this drug).

\section{OPC-67683 (DELAMANID)}

Delamanid is a nitro-dihydro-imidazo-oxazole and is a potent experimental drug against drug resistant and drug susceptible TB disease. It has the same mechanism of action as that of PA-824 (Matsumoto et al., 2006). MIC of OPC-67683 is in the range $0.006-0.024 \mu \mathrm{g} / \mathrm{ml}$ for MTB. This drug prevents the synthesis of a cell wall component called mycolic acid (generally inhibits the synthesis of methoxy- and keto-mycolic acid and hence differentiating it with isoniazid which inhibits all subclasses of mycolic acid) and kills the bacteria by cell wall disruption. In vitro studies \& animal experiments have shown that OPC-67683 is more potent than other first line drugs at smaller concentration with the activity against drug susceptible and drug resistant TB (TB Online, TB Online, OPC-67683; http://www.tbonline.info/posts/2011/9/15/opc67683).

Phase I and II trials of OPC-67683 have been completed and phase III trials are under way. It was observed in phase I trials that doses upto $300 \mathrm{mg}$ was well tolerated by humans with no serious side-effects (Diacon, 2011). Further laboratory and clinical studies are needed to confirm Delamanid's high activity and less toxicity (TB Online, TB Online, OPC-67683; http://www.tbonline.info/posts/2011/9/15/opc67683). 


\section{Linezolid}

Linezolid is a synthetic anti-biotic which belongs to oxazolidinone family (Fig. 1). It was developed by Pharmacia and Upjohn Company and marketed by Pfizer under the brand names Zyvox (in US), Zyvoxid (in Europe) and Zyvoxam (in Canada and Mexico). It is the only oxazolidinone which was approved by FDA in 2000 for the treatment of pneumonia, skin and soft tissue infections caused by gram positive bacteria (Grosset $e t$ al., 2012). It is a protein synthesis inhibitor which hampers survival of bacteria by disrupting translation of messenger RNA into proteins in ribosome. It has a MIC in the range $0.125-1.0 \mu \mathrm{g} / \mathrm{mL}$ for MTB. It is under phase-II clinical trials and it was observed that use of Linezolid improves sputum-culture conversion at 4 month in group of patients receiving immediate versus delayed Linezolid. $89 \%$ patients had sputum-culture conversion on solid medium by 6 month for both kinds of Linezolid regimens (Zumla et al., 2013). Although it is an effective drug in case of MDR-TB and XDR-TB, it shows some severe side-effects on long term administration. Some of the side effects reported are anaemia, thrombocytopenia, peripheral and optic neuropathy in MDR-TB patients (Yew et al., 2008; Migliori et al., 2009).

\section{PNU-100480}

PNU-100480 or Sutezolid is an analogue of Linezolid and is supplied by Pfizer. It has the same MIC as that of Linezolid that is in the range $0.125-1.0 \mu \mathrm{g} / \mathrm{ml}$ for MTB (Grosset et al., 2012; Leach et al., 2007). It shows highly potent bactericidal activity in-vitro and in murine model of TB at lower drug concentration (Williams et al., 2009). PNU-100480 shows activity against both the MTB strains (MDR-TB and XDR-TB). It is under phase IIa trial. To evaluate the safety, tolerability, pharmacokinetics and pharmacodynamics of PNU100480 in a first study, multiple doses upto $1200 \mathrm{mg}$ of it were given to healthy persons for 14 days and $600 \mathrm{mg}$ for 28 days, on the last 2 days of dosing, Pyrazinamide was added. These doses were safe and tolerable (Wallis et al., 2011).

\section{AZD-5847 OR POSIZOLID}

AZD-5847 is a highly modified oxazolidinone developed by Astra Zenecca against TB. MIC of AZD5847 is same as that of Linezolid and PNU-100480. It acts against bacterial protein synthesis and shows activity against drug susceptible MDR-TB and XDR-TB isolates. It was well tolerated and safe when administered in healthy volunteers (2400 mg maximum dose for 14 days) but on higher exposure, reversible changes were observed in white blood cells and reticulocyte counts (Shaw and Barbachyn, 2011). Currently, posizolid is in phase II clinical trials.

\section{$S Q-109$}

SQ-109 is a 1, 2-ethylenediamine ethambutol analogue, under development by Sequella Inc for treatment against TB (Protopopova et al., 2005). It has an MIC of $0.5 \mu \mathrm{g} / \mathrm{mL}$ for MTB (Table 1). SQ-109 acts on cell wall formation by inhibiting trehalose monophosphate transferase while in case of ethambutol, arabinosyl transferase gets inhibited (Tahlan et al., 2012; Takayama et al., 1989), SQ-109 retains potency against EMB resistant strains. SQ-109 has completed phase-I studies in US and first of phase II clinical studies in Africa. In pre-clinical studies, it increased the activity of Isoniazid and Rifampin and shortened the treatment duration by $30 \%$ in murine models. It shows activity against both drug susceptible and MDR-TB including XDR-TB strain (Maxwell Biotech Venture Fund's Portfolio Company).

\section{Vaccines Under Development for Tuberculosis Prevention}

$\mathrm{BCG}$ is the only currently available vaccine for $\mathrm{TB}$. It was used in 1921 for the first time in infants after passing through extensive tests in animals. It is most efficient vaccine in protecting children from TB but its effects in adults is variable. BCG vaccination is included in Expanded Programme of immunization since 1974 (Kaufmann et al., 2010). BCG vaccination is safe in normal population without serious harmful effects but in HIV-infected/immunocompromised individuals, there is high risk of disseminated BCG disease especially in HIVinfected infants (Azzopardi et al., 2009; Hesseling et al., 2007a). Because of this reason and for ensuring safety in HIV-infected infants, WHO's Global Advisory Committee on Vaccine, recommend that BCG should not be administered in such infants (WHO, 2007; Hesseling et al., 2007b). Though BCG has widespread use in new-born babies, it is not effective in preventing adult pulmonary disease, in turn not helping in reduction of global burden of TB disease. Currently some of the strategies for vaccination in progress involve vaccination with viable vaccine, as prime boost or replacement for BCG. But still BCG represents an excellent basis for newly developed prime-boost strategies but in future its replacement has to be considered. To eliminate this deadly disease, vaccinations strategies have to be developed that target dormant MTB and prevent its re-activation. Such novel vaccine strategies are not only effective in children but have proved to be advantageous to adults (Kaufmann et al., 2010). There are 12 vaccines in pipeline which may prove effective in TB prevention in future.

\section{Live Mycobacterial Vaccines}

In the development of novel live mycobacterial vaccines, 2 basic strategies are being employed, first is to improve BCG by adding or over expressing MTB immune-dominant antigens and second is to alter the 
bacteria to be more effective at inducing MTB like responses. Assessment of safety and efficacy of new vaccines that can replace BCG will require vigorous and careful analyses with assays and trials.

\section{$r B C G 30$}

rBCG30 is the future vaccine against TB developed by Marcus A Horwitz's team at University of California, Los Angeles (Kaufmann et al., 2010; Horwitz et al., 2006) (Fig. 2). rBCG30 is genetically modified to produce huge amounts of a $30 \mathrm{kDa}$ antigen (Antigen 85B) that produces strong immune responses both in animals and humans. A very low inoculum of $\mathrm{rBCG} 30$ organisms has the ability to induce strong protective immunity against TB and it is an extremely potent delivery system for mycobacterial antigens (Horwitz et al., 2006). rBCG30 has completed phase I trials that demonstrated it to be a safe immunogenic vaccine (Hoft et al., 2008).

\section{VPM1002}

VPM1002 is an improved vaccine created in Max Planck Institute for Infection Biology in Berlin and is being developed by Vakzine Projekt Management (http://pipelinereport.drupalgardens.com/sites/pipelinere port.drupalgardens.com/files/tb\%20vaccine.pdf),

Tuberculosis vaccine pipeline by Richard Jefferys). VPM1002 is a recombinant strain that was prepared by integrating the gene of Listeria inactivating a gene for Urease $\mathrm{C}$ of $\mathrm{BCG}$. Listeriolysin leads to perforation of phagosome after endocytotic uptake of bacterium, promoting antigen translocation into cytosol and facilitates cross-priming and Th17 cells stimulation through increased apoptosis. Inactivation of gene for Urease C (Ure C) leads to formation of acidic condition which helps in listeriolysin activity. This vaccine has been undergoing clinical trials since 2008. In phase Ia trials conducted in Germany, VPM1002 was effective in preventing TB and fulfilled the safety requirements and the phase Ib trials on infants were conducted to check its safety and tolerability in South Africa (http://scitechdaily.com/vpm1002-tuberculosis-vaccinein-phase-ii-trial/),VPM1002 Tuberculosis Vaccine in Phase II trials, scitechdaily).

\section{MTBVAC01}

MTBVAC01 is the most advanced vaccine designed by TBVI's research partner Carlos Martin, University of Zaragoza, Spain (Table 2). MTBVAC01 is the first and foremost attenuated live vaccine worldwide. This vaccine was prepared by disruption of transcriptional regulation gene phoP and fadD26 (phoP is associated with MTB virulence) (Kaufmann et al., 2010).

\section{Subunit and Live-Vector based Vaccines that Boost BCG Prime}

Subunit vaccines are based on Antigen which is recognized by $\mathrm{T}$-cells, taken from patients with latent infection or from the one who's TB has been cured. Two types of products have been formed; recombinant fusion proteins which consist of two or three dominant MTB or BCG antigens. They can be given repeatedly but an adjuvant is needed that promotes Thl immune responses. On the other hand, live viral vector vaccines are those that express some or several mycobacterial proteins. They do not require adjuvant but can be inhibited by previous exposure to vectors. They trigger Th1 dominated immune response to express heterologous antigen and induce CD-8 T-cell responses (Kaufmann et al., 2010).

\section{H1-IC 31}

H1-IC31 is an adjuvanted subunit vaccine developed by Inter cell, Vienna, Austria (http://pipelinereport.drupalgardens.com/sites/pipelinere port.drupalgardens.com/files/tb\%20vaccine.pdf),

Tuberculosis vaccine pipeline by Richard Jefferys). This vaccine is a fusion of Ag85B protein and ESAT-6 with IC31, a strong Th1 adjuvant. H1-IC31 vaccine induces strong Th1 response against Ag85B protein of MTB. In phase I trials, this vaccine was demonstrated to be safe and well tolerated without any complications and harmful effects. The short-lived response to Early Secretory Antigenic Target-6 (ESAT-6) does not seem to interfere with the ESAT-6 based diagnostic assays done after immunization (Kaufmann et al., 2010).

\section{H1-CAF01}

H1-CAF01 is a recombinant vaccine based on primeboost strategy, formed by fusion of Ag85A-ESAT-6 in CAF01 liposome based adjuvant. H1-CAF01 is under phase I trials (Kaufmann et al., 2010).

\section{H4-IC31 (AERAS-404)}

H4-IC31 is an alternative to H1-IC31 because in this ESAT-6 (fusion protein) was replaced by TB-10.4 to reduce risk of interference with ESAT-6 based diagnostic assays (Fig. 2). Currently H4-IC31 is in phase I trials (Kaufmann et al., 2010).

\section{H56-IC31}

It is also a recombinant protein vaccine which has Rv2660 as an additional ingredient along with antigen 85B, ESAT- 6 and the IC31 adjuvant. The trial for SSI H56-IC31 vaccine is being conducted in collaboration with Aeras and is supported by the Bill \& Melinda Gates Foundation Grand Challenge \#12 consortium, (http://pipelinereport.drupalgardens.com/sites/pipelinere port.drupalgardens.com/files/tb\%20vaccine.pdf), Tuberculosis vaccine pipeline by Richard Jefferys). 
Table 1. General description of new drugs

\begin{tabular}{|c|c|c|c|}
\hline Drugs & Company & $\begin{array}{l}\text { Minimum Inhibitory } \\
\text { Concentration (MIC) } \\
\text { in } \mu \mathrm{g} / \mathrm{mL}\end{array}$ & Indications \\
\hline Rifapentine & Sanofi aventis & 0.06 & $\begin{array}{l}\text { Pulmonary TB but sometimes used in MDR-TB } \\
\text { patients which are resistant to rifampicin }\end{array}$ \\
\hline Clofazimine & Novartis & $0.6-1.2$ & MDR-TB and XDR-TB (Van et al., 2010) \\
\hline Moxifloxacin & Bayer AG & 0.5 & $\begin{array}{l}\text { Pulmonary TB and } \\
\text { MDR-TB }\end{array}$ \\
\hline TMC-207 & Johnson and Johnson & 0.06 & MDR-TB (Diacon et al., 2007) \\
\hline PA-824 & TB Alliance and Novartis & 0.125 & $\begin{array}{l}\text { Drug susceptible as well as drug resistant TB } \\
\text { (i.e., MDR-TB) }\end{array}$ \\
\hline OPC-67683 & Otsuka Pharmaceutical Co. Ltd. & $0.006-0.024$ & $\begin{array}{l}\text { Drug susceptible as well as drug resistant TB } \\
\text { (i.e., MDR-TB) }\end{array}$ \\
\hline Linezolid & Pfizer & $0.125-1.0$ & MDR-TB and XDR-TB (Diacon, 2011; Zumla et al., 2013) \\
\hline PNU-100480 & Sequella Inc. & $0.125-1.0$ & MDR-TB and XDR-TB \\
\hline AZD-5847 & Astra Zenecca & $0.125-1.0$ & MDR-TB and XDR-TB \\
\hline SQ-109 & Sequella Inc. & 0.5 & MDR-TB and XDR-TB \\
\hline
\end{tabular}

Table 2. General description of new vaccines

\begin{tabular}{|c|c|c|c|}
\hline Vaccine & Type & Strategy & Funders \\
\hline rBCG30 & Recombinant live & Prime & $\begin{array}{l}\text { University of California, Los Angeles, Aeras } \\
\text { (Takayama and Kilburn, 1989) }\end{array}$ \\
\hline VPM1002 & Recombinant live & Prime & $\begin{array}{l}\text { Vakzine Projekt Management, Max Planck Institute, Tuberculosis } \\
\text { Vaccine Initiative (Hesseling et al., 2007a) }\end{array}$ \\
\hline H1-IC31 & Recombinant protein & Prime-boost & $\begin{array}{l}\text { Statens Serum Institute, Tuberculosis Vaccine Initiative, Intercell } \\
\text { (Takayama and Kilburn, 1989) }\end{array}$ \\
\hline H1-CAF01 & Recombinant protein & Prime-boost & $\begin{array}{l}\text { Statens Serum Institute, Tuberculosis Vaccine Initiative } \\
\text { (Takayama and Kilburn, 1989) }\end{array}$ \\
\hline H4-IC3 1 & Recombinant protein & Prime-boost & $\begin{array}{l}\text { Statens Serum Institute, Aeras, Sanofi, Intercell } \\
\text { (Takayama and Kilburn, 1989; Hesseling et al., 2007b) }\end{array}$ \\
\hline H56-IC31 & Recombinant protein & Prime-boost & $\begin{array}{l}\text { Statens Serum Institute, Aeras, Bill and Melinda Gates } \\
\text { Foundation (Hesseling et al., 2007a) }\end{array}$ \\
\hline GSK-M72 & Recombinant protein & Prime-boost & $\begin{array}{l}\text { Glaxo Smith Kline, Aeras, Tuberculosis Vaccine Initiative } \\
\text { (Takayama and Kilburn, 1989) }\end{array}$ \\
\hline $\operatorname{AdAg} 85 \mathrm{~A}$ & Viral & Prime & Can Sino Biotechnology Inc, Aeras (Hesseling et al., 2007b) \\
\hline Crucell Ad35 & Viral vector & Prime-boost & Crucell N. V., Aeras (Hesseling et al., 2007a) \\
\hline MVA85A & Viral vector & Prime-boost & $\begin{array}{l}\text { Wellcome Trust, Aeras, Oxford-Emergent Tuberculosis Consortium } \\
\text { (Takayama and Kilburn, 1989; Hesseling et al., 2007b) }\end{array}$ \\
\hline RUTI & Fragmented MTB & Immunotherapeutic & Archivel Farma (Hesseling et al., 2007a) \\
\hline
\end{tabular}

\section{GSK M72}

GSK M72 is a recombinant protein which is formulated in a vaccine adjuvant system containing a liposomal preparation and immunostimulants MPL and QS21. Phase I trials for GSK M72 vaccine has been completed and demonstrated it to be tolerable and potent vaccine in inducing polyfunctional CD4 T-cell responses against M72 antigens in healthy adults that have persisted for atleast 3 years, (http://pipelinereport.drupalgardens.com/sites/pipelinere port.drupalgardens.com/files/tb\%20vaccine.pdf), Tuberculosis vaccine pipeline by Richard Jefferys).

\section{AdAg85A and Crucell Ad35 (AERAS-402)}

Both of these vaccines are replication deficient (E1 deleted) recombinant adenoviruses. AdAg85A is different from AERAS-402 as it expresses only Ag85A but AERAS-402 expresses Ag85A, Ag85B and TB-10.4 as well (http://pipelinereport.drupalgardens.com/sites/pipelinere port.drupalgardens.com/files/tb\%20vaccine.pdf),

Tuberculosis vaccine pipeline by Richard Jefferys). These vaccines are helpful in inducing CD-8 T-cell responses. But several studies had show that natural exposure to homologous or cross-reacting strain of adenovirus can interfere with adenovirus based vaccine responses (Kaufmann et al., 2010).

\section{MVA85A}

MVA85A (Modified Vaccinia Ankara 85A) is a new generation recombinant vaccine which is a replication deficient vaccinia virus that expresses Ag85A (Kaufmann et al., 2010) (Table 2). Phase I clinical trials have been completed. In initial trials, it was observed that MVA85A vaccine boosted previous immunity and induced strong Th1 responses but phase II b trials in infants proved to be disappointing as the expected protection was seen to be very low in infants (BBC News, Tuberculosis Vaccine Hopes Dashed). 


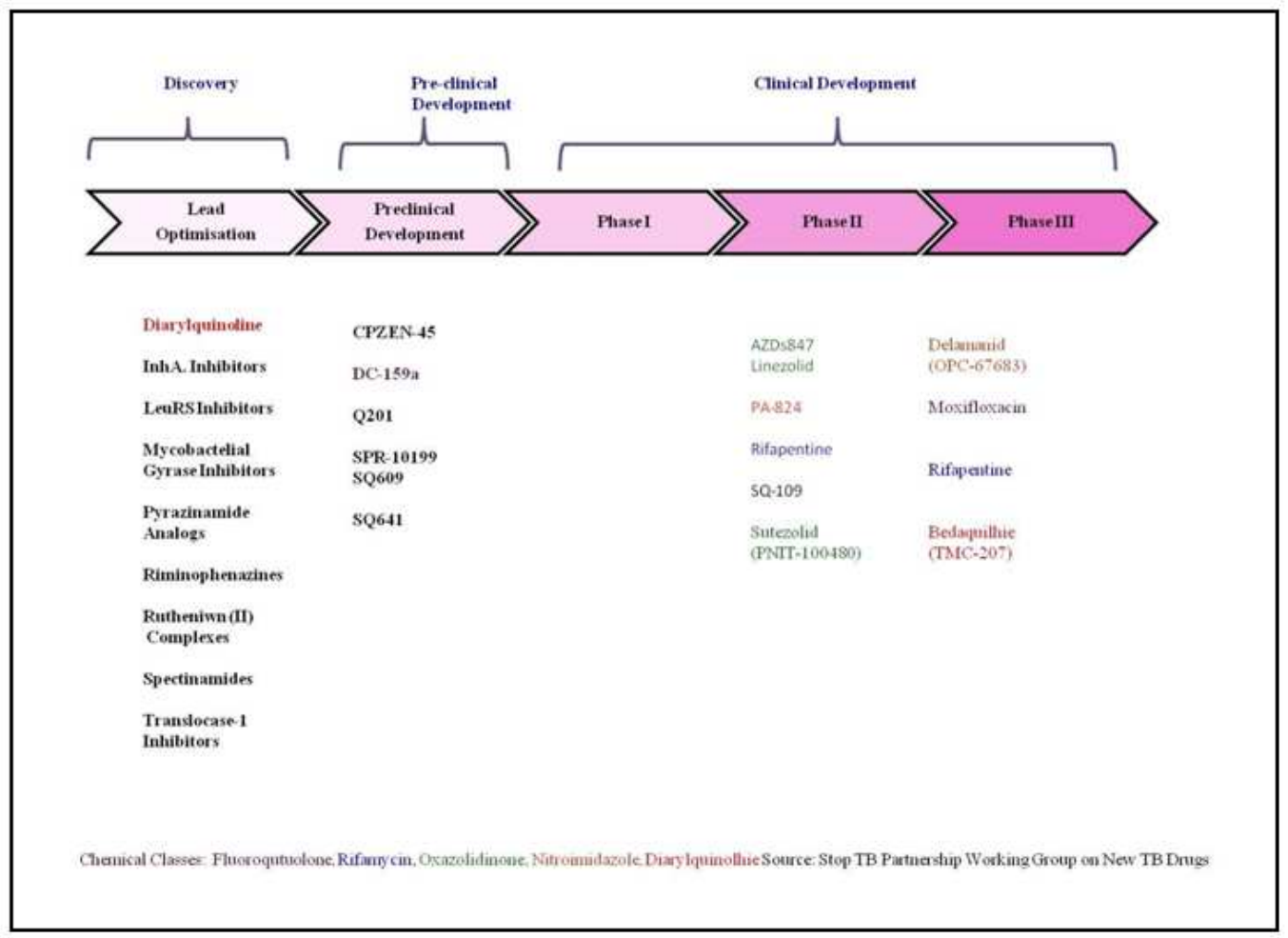

Fig. 1. New TB drugs in pipeline

TB Vaccine Pipeline

\begin{tabular}{|l|l|r}
\hline Phase I & \multicolumn{1}{|c|}{ Phase lla } & Phase \\
\hline AdAg85A & GSK-M72 & MVA85A \\
(P)(B) & (B) PI) & (B) (PI) (II)
\end{tabular}

$\begin{array}{lll}\text { H1-CAF01 } & \text { VPM-1002 } & \text { Crucell Ad35 } \\ \text { (P)(B)(PI) } & \text { (P)B } & \text { (B) }\end{array}$

$\begin{array}{ll}\text { H56-IC31 } & \text { H1-IC31 } \\ \text { (P)(B) } & \text { (P) (B) PPI) }\end{array}$

H4-IC31 RUTI

(B) (B) (PI)

Source: Tuberculosis Vaccine Candidates-2011

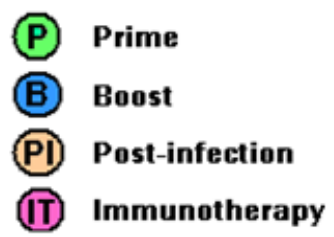

Fig. 2. New TB vaccine pipeline 


\section{RUTI}

RUTI is produced from cell fragments of detoxified MTB and delivered in liposomes (Kaufmann et al., 2010; Cardona, 2006). RUTI is an immunotherapeutic (Table 2) vaccine which facilitates a balanced $\mathrm{T}$ helper response to a wide range of antigens along with heavy production of antibody. RUTI was originally discovered in Institute Germans i Pujol but now it is being developed by Biotech Company Archivel Farma. In phase II clinical trials, different doses of this vaccine were well tolerated in both HIV negative as well as HIV positive adults. RUTI is highly active in inducing long term memory $\mathrm{T}$-cell responses against multiple TB antigens. Currently RUTI is in phase-III clinical trials and soon will be available for human use (Press Release; Archivel secures funding to bring the antituberculosis therapeutic vaccine RUTI to market; The TB Vaccines Pipeline-TBVI,

http://www.tbvi.eu/fileadmin/user_upload/Documenten/ $\mathrm{N}$ ews/TBVaccines_pipeline_report_TAG_1July2013.pdf)

\section{Conclusion}

TB is a contagious infectious disease caused by MTB, an aerobic pathogenic bacterium that usually infects lungs. At present, it has been estimated that one third of world population is infected with tubercle bacilli which is responsible for 8-10 million new cases of TB and 3 million deaths annually throughout the world. It is very challenging to eradicate this disease due to emergence of MDR-TB and XDR-TB; moreover co-infection with HIV increases the cruelty of this disease. New drugs and vaccines are immediately required for eliminating this life threatening disease for achieving the goal of TB elimination. The above mentioned drugs and vaccines are carefully assessed in clinical trials for their safety in various groups of human population. Several research groups and institutions worldwide are working together to create new vaccines and drugs that will overcome the challenges posed by active TB disease. For advances in drug and vaccine discovery, there is need of funding, community acceptance and advocacy. Emphasis on new, creative approaches and support by social and political groups may lead to elimination of TB from human society.

\section{Future Prospective}

New anti-tuberculosis drugs offer hopes of shortened treatment regimens for drug susceptible, drug resistant and latent infection. The quest for better vaccines for controlling tuberculosis among human population in future continues. For the eradication of TB, several research groups are working on Mycobacterium tuberculosis with the goal of TB elimination. We should emphasize on new drug discovery by creative approaches which will eliminate TB from human population.

\section{Acknowledgement}

We thank Dr. Rajesh S. Gokhale for making this work possible. The authors acknowledge financial support from GAP0092 of the Department of Science and Technology and Council of Scientific and Industrial Research.

\section{Author's Contributions}

Jaishree Meena: The author contributes in drafting the article or reviews it critically for significant intellectual content.

Laxman S. Meena: The author contributes in conception and design and/ or acquits the data and/or analysis of interpret the data.

\section{Ethics}

Authors should address any ethical issues that may arise after the publication of this manuscript.

\section{References}

http://pipelinereport.drupalgardens.com/sites/pipelinerep ort.drupalgardens.com/files/tb\%20vaccine.pdf), Tuberculosis vaccine pipeline by Richard Jefferys.

http://scitechdaily.com/vpm1002-tuberculosis-vaccinein-phase-ii-trial/

Andries, K., P. Verhasselt, J. Guillemont, H.W. Gohlmann and J.M. Neefs et al., 2005. A diarylquinoline drug active on the ATP synthase of Mycobacterium tuberculosis. Science, 307: 223-227. DOI: 10.1126/science.1106753

Arioli, V., M. Berti, G. Carniti, E. Randisi and E. Rossi et al., 1981. Antibacterial activity of DL 473, a new semisynthetic rifamycin derivative. J. Antibiot., 34: 1026-1032. DOI: 10.7164/antibiotics.34.1026

Azzopardi, P., C.M. Bennett, S.M. Graham and T. Duke, 2009. Bacille Calmette-Guerin vaccine-related disease in HIV-infected children; A systematic review. Int. J. Tuberc. Lung. Dis., 13: 1331-44. PMID: 19861003

Barry, V.C., J.G. Belton, M.L. Conalty, J.M. Denneny and D.W. Edward et al., 1957. A new series of phenazine (rimino-compounds) with high antituberculosis activity. Nature (London)., 179: 1013-1015. DOI: $10.1038 / 1791013 \mathrm{a} 0$

Barry, V.C., M.L. Conalty and E.E. Gaffney, 1956. Anti tuberculosis activity in the phenazine series. Isomeric pigments obtained by oxidation of $\mathrm{O}-$ Phenylenediamine derivatives. J. Pharm. Pharmacol., 8: 1089-1096. DOI: $10.1111 / \mathrm{j} .2042-7158.1956 . t b 12238 . \mathrm{x}$

BBC News, Tuberculosis Vaccine Hopes Dashed. http://www.bbc.com/news/health-21302518 
Cardona, P.J., 2006. RUTI: A new chance to shorten the treatment of latent tuberculosis infection. Tuberculosis, 86: 273-289. DOI: 10.1016/j.tube.2006.01.024

Cole, S.T. and P.M. Alzari, 2005. TB: A new target, a new drug. Science, 307: 214-215.

DOI: $10.1126 /$ science. 1108379

Conde, M.B., A. Efron, C. Loredo, G.R.M. De Souza and N.P. Graca et al., 2009. Moxifloxacin versus ethambutol in the initial treatment of tuberculosis: A double-blind, randomised, controlled phase II trial. Lancet, 373: 1183-1189.

DOI: 10.1016/S0140-6736(09)60333-0

Diacon, A.H., A. Pym, M. Grobusch, R. Patientia and R. Rustomjee et al., 2007. The diarylquinoline TMC 207 for Multidrug-resistant tuberculosis. N. Engl. J. Med., 360: 2397-405. DOI: 10.1056/NEJMoa0808427

Diacon, A.H., 2011. Early bactericidal activity of delamanid (OPC-67683) in smear positive pulmonary tuberculosis patients. Int. J. Tuberc. Lung. Dis., 15: 949-954.

Dorman, S.E., J.L. Johnson, S. Goldberg, G. Muzanye and N. Padoyatchi et al., 2009. Tuberculosis Trials Consortium, Substitution of moxafloxacin for isoniazid during intensive phase treatment of pulmonary tuberculosis. Am. J. Respir. Crit. Care. Med., 180: 273-280.

Fact Sheet: Why Bedaquiline (TMC207) should be prioritised for drug-resistant TB patients in South Africa. www.msfaccess.org/.../fact-sheet-whybedaquiline-tmc207-should-be-prioritised-drugresistant-tb-patients-south

Grosset, J.H., T.G. Singer and W.R. Bishai, 2012. New drugs for the treatment of Tuberculosis: Hope and reality. Int. J. Tuberc. Lung. Dis., 16: 1005-1014.

Grumbach, E., 1960. Activite antituberculeuse experimentale de deux derives de phenazine pigmentee (B663 et 720) seuls et associes a' d'autres antituberculeux (isoniazide et ethioniamide). Ann. Inst. Pasteur., 99: 567-585.

Hesseling, A.C., M.F. Cotton, B.J. Marais, R.P. Gie and H.S. Schaaf et al., 2007a. BCG and HIV reconsidered: Moving the research agenda forward. Vaccine, 25: 6565-6568.

Hesseling, A.C., B.J. Marais, R.P. Gie, H.S. Schaaf and P.E. Fine et al., 2007b. The risk of disseminated Bacille-Calmette-Guerin (BCG) disease in HIVinfected children. Vaccine, 25: 14-18.

Hoft, D.F., A. Blazevic, G. Abate, W.A. Hanekam and G. Kaplan et al., 2008. A new recombinant BacilleCalmette-Guerin vaccine safely induces significantly enhanced TB-specific immunity in human volunteers. J. Infect. Dis., 198: 1491-1501.

Horwitz, M.A., G. Harth, B.J. Dillon and S. MaslesaGalić, 2006. Extraordinarily few organisms of a live recombinant $\mathrm{BCG}$ vaccine against tuberculosis induce maximal cell-mediated and protective immunity. Vaccine, 24: 443-451.
Ji, B., N. Lounis, C. Maslo, P.C. Truffot and P. Bonnafous et al., 1998. In vitro and in-vivo activities of moxafloxacin and clinafloxacin against Mycobacterium tuberculosis. Antimicrob. Agents. Chemother, 42: 2066-2069.

Kaufmann, S.H.E., G. Hussey and P.H. Lambert, 2010. New vaccines for Tuberculosis. Lancet, 375: 2110-2119.

Koul, A., N. Dendouga, K. Vergauwen, B. Molenberghs and L. Vranckx et al., 2007. Diarylquinolines target subunit $\mathrm{c}$ of mycobacterial ATP synthase. Nat. Chem. Biol., 3: 323-324.

Leach, K.L., Swaney, S.M and J.R. Colca, 2007. The site of action of oxazolidinone antibiotics in living bacteria and in human mitochondria. Mol. Cell., 26: 393-402.

Lenaerts, A.J., V. Gruppo, J.V. Brooks and I.M. Orme, 2003. Rapid in vivo screening of experimental drugs for tuberculosis using gamma interferon gene-disrupted mice. Antimicrob. Agents. Chemother., 47: 783-785.

Lenaerts, A.J., V. Gruppo, H.S. Marietta, C.M. Johnson and D.K. Driscole et al., 2005. Preclinical testing of the nitroimidazopyran PA-824 for activity against Mycobacterium tuberculosis in a series of in vitro and in vivo models. Antimicrob. Agents. Chemother, 49: 2294-2301.

Matsumoto, M., H.H. Hiroyuki, T. Tomishige, M. Kawasaki and H. Tsobouchi et al., 2006. OPC67683, a nitro-dihydro-imidazooxazole derivative with promising action against tuberculosis in vitro and in mice. PLoS. Med., 3: 466.

Maxwell Biotech Venture Fund's Portfolio Company, Infected, receives Russian Regulator's Approval to conduct Pivotal Clinical Trial for Sequella's Antibiotic SQ-109 for Tuberculosis. SQ-109 will be evaluated for efficacy on multidrug resistant patients.

Meena, L.S., P. Chopra, R.S. Bedwal and Y. Singh, 2008. Cloning and Characterization of a GTP binding protein from $M$. tuberculosis $\mathrm{H}_{37} \mathrm{Rv}$. Enzyme. Microb. Technol., 42: 138-144.

Meena, L.S., P. Chopra, R.A. Vishwakarma and Y. Singh, 2013. Biochemical characterization of an Sadenosyl-L-methionine dependent methyltransferase of Mycobacterium tuberculosis. Biol. Chem., 394: 871-877.

Meena, L.S and Rajni, 2011. Cloning and Characterization of engA, a GTP-binding protein from Mycobacterium tuberculosis $\mathrm{H}_{37} \mathrm{Rv}$. Biologicals, 39: 94-99.

DOI: $10.1016 /$ j.biologicals.2011.01.005

Meena, L.S and Rajni, 2010. Survival mechanisms of pathogenic Mycobacterium tuberculosis $\mathrm{H}_{37} \mathrm{Rv}$. FEBS. J., 277: 2416-2427. DOI: 10.1111/j.17424658.2010.07666.x

Migliori, G.B., B. Eker, M.D. Richardson, G. Sotgiu and J.P. Zellweger et al., 2009. Retrospective TBNET assessment of linezolid safety, tolerability and efficacy in multidrug resistant tuberculosis. Eur. Respir. J., 34: 387-393. 
Miyazaki, E., M. Miyazaki, J.M. Chen, R.E. Chaisson and W.R. Bishai, 1999. Moxifloxacin (BAY128039), a new 8-methoxyquinolone, is active in a mouse model of tuberculosis. Antimicrob. Agents. Chemother., 43: 85-89. PMID: 9869570

Nuermberger, E., I. Rosenthal, S. Tyagi, K. Williams and D. Almeida et al., 2006. Combination chemotherapy with the nitroimidazopyran PA-824 and first-line drugs in a murine model of tuberculosis. Antimicrob Agents Chemother, 50: 2621-2625. PMID: 16870750

Nuermberger, E., S. Tyagi, R. Tasneen, K. Williams and D. Almeida et al., 2008. Powerful bactericidal and sterlizing activity of a regimen containing PA-824, moxafloxacin and pyrazinamide in a murine model of tuberculosis. Antimicrob. Agents. Chemother., 52: 1522-1524.

Nuermberger, E.L., T. Yoshimatsu, S. Tyagi, R.J. O'Brien and A.N. Vernon et al., 2004. Moxifloxacin-containing regimen greatly reduces time to culture conversion in murine tuberculosis. Am. J. Respir. Crit. Care. Med., 169: 421-426. PMID: 14578218

Press Release; Archivel secures funding to bring the antituberculosis therapeutic vaccine RUTI to market.

Protopopova, M., C. Hanrahan, B. Nikonenko, R. Samala and P. Chen et al., 2005. Identification of a new anti-tubercular drug candidate, SQ-109, from a combinatorial library of 1, 2-ethylenediamines. J. Antimicrob. Chemother., 56: 968-974.

Rajni and L.S. Meena, 2010. Guanosine triphosphatases as novel therapeutic targets in tuberculosis. Inter. J. Infect. Dis., 14: e682-e687. DOI: $10.1016 /$ j.ijid.2009.11.016

Rajni, N. Rao and L.S. Meena, 2011. Biosynthesis and toxic behavior of lipids produced by Mycobacterium tuberculosis: LAM and Cord factor: An overview. Biotechnol. Res. Inter. DOI: $10.4061 / 2011 / 274693$

Rustomjee, R., C. Lienhardt, T. Kanyok, G.R. Davies and D.J. Coleman et al., 2008. Gatifloxacin for TB (OFLOTUB) study team. A Phase II study of the sterilizing activities of ofloxacin, gatifloxacin and moxafloxacin in pulmonary tuberculosis. Int. J. Tuberc. Lung. Dis., 12: 128-138.

Shaw, K.J. and M.R. Barbachyn, 2011. The oxazolidinones: Past, present and future. Ann. N.Y. Acad. Sci., 1241: 48-70. DOI: 10.1111/j.1749-6632.2011.06330.x

Singh, R., A.R. Meena and L.S. Meena, 2011. Multidrug resistant-TB and extensively drug resistant-TB: A nuisance to medical science. J. Bacteriol. Parasitol., 2: 1-5.

Stover, C.K., P. Warrener, D.R. van Devanterr, D.R. Sherman and T.M. Arain et al., 2000. A small molecule nitroimidazopyran drug candidate for the treatment of tuberculosis. Nature, 405: 962-966.
Tahlan, K., R. Wilson, D.B. Kastrinsky, K. Arora and V. Nair et al., 2012. SQ-109 targets Mmpl3, a membrane transporter of trehalose monomycolate involved in mycolic acid donation to the cell wall core of Mycobacterium tuberculosis. Antimicrob. Agents. Chemother., 56: 1797-1809.

Takayama, K. and J.O. Kilburn, 1989. Inhibition of synthesis of arabinogalactan by ethambutol in Mycobacterium smegmatis. Antimicrob. Agents. Chemother, 33: 1493-1499.

Tasneen, R., S. Tyagi, K. Williams, J.H. Grosset and E. Nuermberger, 2008. Enhanced bactericidal activity of rifampin and/or pyrazinamide when combined with PA-824 in a murine model of tuberculosis. Antimicrob. Agents. Chemother, 52: 3664-3668.

TB Drugs; http://www.tbfacts.org/tb-drugs.html

TB Online, Moxifloxacin; www.tbonline.info/posts/2011/8/23/moxifloxacin, OPC-67683;

http://www.tbonline.info/posts/2011/9/15/opc67683

The TB Vaccines Pipeline-TBVI,

http://www.tbvi.eu/fileadmin/user_upload/Documen ten/News/TBVaccines_pipeline_report_TAG_1July 2013.pdf

The TB Vaccines Pipeline-TBVI. http://www.tbvi.eu/fileadmin/user_upload/Documen ten/News/TBVaccines_pipeline_report_TAG_1July 2013.pdf

Tyagi, S., E. Nuermberger, T. Yoshimatsu, K. Williams and I. Rosenthal et al., 2005. Bactericidal activity of the nitroimidazopyran PA824 in a murine model of tuberculosis. Antimicrob. Agents. Chemother., 49: 2289-2293.

Van, D.A., A.K. Maug, M.A. Salim, P.K. Das and M.R. Sarker et al., 2010. Short, highly effective and inexpensive standardized treatment of multidrug resistant tuberculosis. Am. J. Respir. Crit. Care. Med., 182: 684-692.

Velayati, A.A., P. Farnia and M.R. Masjedi, 2013. The Totally Drug Resistant Tuberculosis (TDR-TB). Int. J. Clin. Exp. Med., 6: 307-309.

Voelker, R., 2013. MDR-TB has new drug foe after fasttrack approval. JAMA, 309: 430-430. DOI: 10.1001/jama.2013.94

VPM1002 Tuberculosis Vaccine in Phase II trials, scitechdaily.

Wallis, R.S., W.M. Jakubiec, V. Kumar, G. Bedarida and A. Silvia et al., 2011. Biomarker-assisted dose selection for safety and efficacy in early development of PNU-100480 for tuberculosis. Antimicrob. Agents. Chemother., 55: 567-574.

WHO, 2012. WHO Global tuberculosis report. http://www.who.int/tb/publications/global_report/gt br12_main.pdf

WHO, 2007. Revised BCG vaccination guidelines for infants at risk for HIV infection. Wkly. Epidemiol. Rec., 82: 193-196. PMID: 17526121 
Williams, K.N., C.K. Stover, T. Zhu, R. Tasneen and S. Tyagi et al., 2009. Promising anti-tuberculosis activity of the oxazolidinone PNU-100480 relative to that of linezolid in a murine model. Antimicrob. Agents. Chemother., 53: 1314-1319.

$\mathrm{Xu}$, H.B., Jiang and H.P. Xiao, 2012. Clofazimine in the treatment multidrug-resistant tuberculosis. Clin. Microbiol. Infect., 18: 1104-1110.

Yawalkar, S. J and W. Vischer, 1979. Lamprene (clofazimine) in leprosy. Basic information. Lepr. Rev., 50: 135-144.
Yew, W.W., Chau, C.H and K.H. Wen, 2008. Linezolid in the treatment of difficult multidrug-resistant tuberculosis. Int. J. Tuberc. Lung. Dis., 12: 345-346.

Yoshimatsu, T., Nuermberger, E., Tyagi, S., Chaisson, R., Bishai, W and J. Grosset, 2002. Bactericidal activity of increasing daily and weekly doses of moxifloxacin in murine tuberculosis. Antimicrob. Agents. Chemother., 46: 1875-1879.

Zumla, A., Raviglione, M., Hafner, R and C.F.V. Reyn, 2013. Tuberculosis; current concepts; N.E.J.M., 368: 745-755. 